\title{
Evaluation of Tobacco Germplasm for Seedling Resistance to Stem Rot and Target Spot Caused by Thanatephorus cucumeris
}

\author{
P. E. Elliott and R. S. Lewis, Department of Crop Science, H. D. Shew and W. A. Gutierrez, Department of Plant \\ Pathology, and J. S. Nicholson, Department of Crop Science, North Carolina State University, Raleigh 27695
}

\begin{abstract}
Elliott, P. E., Lewis, R. S., Shew, H. D., Gutierrez, W. A., and Nicholson, J. S. 2008. Evaluation of tobacco germplasm for seedling resistance to stem rot and target spot caused by Thanatephorus cucumeris. Plant Dis. 92:425-430.

Stem rot and target spot of tobacco, caused by Rhizoctonia solani and its teleomorph Thanatephorus cucumeris, respectively, can cause serious problems in production of tobacco (Nicotiana tabacum) seedlings. Previous screens for genetic resistance in tobacco have been limited. The objective of this study was to evaluate 97 genotypes composing several classes of tobacco and related Nicotiana spp. for seedling resistance to stem rot and target spot. Significant differences in disease incidence initially were observed among the genotypes for both stem rot and target spot; however, resistance to target spot was not observed when disease pressure was high. Partial resistance to stem rot was observed in several genotypes in repeated tests. These accessions may be useful as a source of resistance to $R$. solani in future breeding efforts.
\end{abstract}

Rhizoctonia solani J.G. Kühn (teleomorph Thanatephorus cucumeris (A.B. Frank) Donk) can cause serious disease problems in both transplant and field production of tobacco. This pathogen became more of a problem on seedlings in the 1990s as tobacco growers largely switched from producing transplants in outdoor plant beds to production in greenhouse float systems. In this system, tobacco is seeded on a soilless growth medium in polystyrene trays which then are floated on a reservoir containing a nutrient solution (18). This produces transplants with very good quality, but the high plant density, high humidity, and shared water reservoir associated with this type of production is very favorable for disease development (17). Reused polystyrene float trays also can harbor $R$. solani sclerotia and hyphae from previous years and can be a primary source of inoculum (5). The fungicide mancozeb (Dithane DF) has been labeled for use to control damping off and target spot in greenhouse production and provides moderate control of these diseases. However, in a comparison of the activity of several fungicides against $R$. solani, it was demonstrated that mancozeb was the least active of the fungicides tested (3). In this test, iprodione provided excellent control for stem rot and target spot symptoms in seed beds but is not currently registered for

Corresponding author: J. S. Nicholson

E-mail: Jennifer.S.Nicholson@aphis.usda.gov

Accepted for publication 19 October 2007.

doi:10.1094/PDIS-92-3-0425

(C) 2008 The American Phytopathological Society use in tobacco greenhouses. Currently recommended controls include proper disinfestation of float trays by steam treatments or methyl bromide before reuse and removal of plant debris near tobacco greenhouses (5). Growers also may choose to use new float trays each year, although this does increase transplant production costs.

$R$. solani causes damping-off and stem rot in young transplants and a disease of the lower stem and root called sore shin in older field plants $(7,15,19)$. This disease can result in seedling death in the greenhouse and moderate to severe stunting and death of plants in the field. Transplanting infected seedlings is a major cause of sore shin in field plants; however, infection also can be caused by $R$. solani that is already present in the field.

Target spot is a relatively new foliar disease that first appeared in the United States in the 1980s and is now an economically important disease in tobacco production $(8,14)$. This disease is caused by infection with basidiospores of $T$. cucumeris which are produced on hymenia that form on the soil surface or infected plant tissue $(14,16)$. Symptoms begin as small water-soaked lesions on the leaves which can expand to large circular spots with concentric rings. This disease can occur on tobacco seedlings in greenhouse environments and usually is observed after the leaves of the seedlings have grown close enough to form a canopy, forming a high-humidity environment that favors disease development. In severe cases, the pathogen may grow from the leaf tissue into the stem, resulting in plant death. Plants with leaf lesions that are transplanted into the field also may provide an inoculum source for later infec- tion, because a second cycle of target spot can occur on large field plants (17). The disease develops after the leaves are large enough to shade the soil, which provides an area of high relative humidity and promotes the production of basidiospores from hymenia on the soil surface. The spores are dispersed onto the lower leaves of the plants and the disease progresses up the plant. Isolates of $R$. solani associated with stem and root rot symptoms have been characterized by anastomosis group as AG-1, AG-2-2, and AG-4, whereas target spot in the greenhouse and the field is associated with AG-3 (20).

Resistance to $R$. solani in tobacco cultivars would be highly desirable but previous screens for resistance in tobacco germplasm have been limited to a small number of commonly used cultivars. However, evaluation of germplasm and identification of resistance to $R$. solani has been successful in other crops such as peanut, bean, rice, sorghum, and sugar beet (4,912). The United States Nicotiana germplasm collection maintained by North Carolina State University contains approximately $1,900 \mathrm{~N}$. tabacum accessions as well as 59 Nicotiana spp., and is a valuable resource for the discovery of new traits (6).

The objective of this work was to screen a diverse sample of the accessions in the Nicotiana collection as well as several elite cultivars representing various market types in order to identify potential sources of seedling resistance to both target spot and stem rot. Improved seedling resistance would reduce direct greenhouse losses due to these diseases. This could reduce crop losses as well by reducing the potential of transplanting infected seedlings into the field.

\section{MATERIALS AND METHODS}

Plant material. Ninety-seven accessions chosen to represent a wide range of Nicotiana spp. diversity were evaluated for resistance. The $N$. tabacum accessions are categorized according to market types and included 2 cigar binder, 11 cigar filler, 11 cigar wrapper, 14 burley, 26 flue-cured, 16 oriental, and 1 each of dark, Maryland, and air-cured types (Table 1). In addition, 14 Nicotiana spp. were evaluated. Accessions chosen were a set of tobacco genotypes collected from around the world, and included popular U.S. cultivars and a selection of tobacco introductions (TIs). The 
TIs were chosen based on diversity of geographic origin. These accessions were obtained from the U.S. Nicotiana Germplasm Collection maintained in Oxford, NC.

Inoculum production. An AG-3 target spot isolate of T. cucumeris and an AG-4 stem rot isolate of $R$. solani were isolated from flue-cured tobacco in North Carolina. The isolates were grown on potato dextrose agar at room temperature (22 to $25^{\circ} \mathrm{C}$ ) for 5 days. Colonized agar plugs were removed and transferred to $250-\mathrm{ml}$ Erlenmeyer flasks containing autoclaved rice grains (16). The pathogen was allowed to thoroughly colonize the rice grains for approximately 2 weeks at room temperature prior to use in experiments.

Inoculation and disease evaluation. Experiments were conducted in walk-in growth chambers in the North Carolina State University Southeastern Plant Environmental Laboratory (Phytotron), Raleigh.

The initial resistance screen was conducted in a randomized complete block design with four replications for the target spot experiment and three replications for the stem rot experiment. Because of space limitations, only one replication (24 seedlings per accession) could be placed into a phytotron chamber at one time. Therefore, the experiment was replicated over time.

The tobacco accessions were germinated and grown in Carolina's Choice tobacco potting mix (Carolina Soil Company, Kinston, NC). For each accession, 2-week-old seedlings were transferred to a 30-cell section of a polystyrene tray ( 5 by 6 cells, each cell 2.3 by 2.3 by $6.2 \mathrm{~cm}$ ) that was cut from a 288-cell float tray from Carolina Soil Company. Each tray served as a replication, with 24 plants and six evenly spaced cells that were left unplanted for introduction of the inoculum. Transplanted trays were floated in polyethylene pans filled with a fertilizer solution of Peters 20-20-20 with nitrogen at $150 \mathrm{ppm}$ to mimic the greenhouse float-tray system used by tobacco growers. Transplants were grown with a 12 -h photoperiod at $28^{\circ} \mathrm{C}$ day and $20^{\circ} \mathrm{C}$ night temperatures. Relative humidity levels in the chamber were typically between 70 and $85 \%$. Distilled water was added to the pans as needed.

Tobacco seedlings were inoculated with either the stem rot or target spot pathogen 2 days after transplanting. Infested rice (approximately six grains) was placed onto the surface of the soil in each of the six unplanted cells and the pathogen was allowed to colonize the trays. Some plants were bordered by more than one inoculation site (cell), but no differences in the amount of disease were observed on the plants in those cells compared with other plants. Plants were evaluated for disease symptoms starting at 1 week after inoculation. Two phases of disease development for both diseases were observed. For the
Table 1. Reaction of Nicotiana accessions to stem rot isolate of Rhizoctonia solani

\begin{tabular}{|c|c|c|c|c|}
\hline Genotype name & PI number ${ }^{\mathrm{a}}$ & Type $^{\text {b }}$ & $\begin{array}{c}\text { Arcsine } \\
\text { incidence }(\%)^{\mathrm{c}}\end{array}$ & AUDPC $^{d}$ \\
\hline TI 1316 & PI 405604 & $\mathrm{CF}$ & 28.7 & 162.1 \\
\hline TI 1311 & PI 239655 & $\mathrm{O}$ & 39.7 & 264.5 \\
\hline Nicotiana rustica & PI 555554 & Sp. & 40 & 217 \\
\hline TI 428 & PI 112830 & $\mathrm{CF}$ & 41.8 & 130.4 \\
\hline Burley 21 & PI 552363 & B & 42.3 & 181.4 \\
\hline TI 1029 & PI 119162 & $\mathrm{CF}$ & 42.7 & 198.8 \\
\hline L8 & PI 551280 & $\mathrm{~B}$ & 42.7 & 121.9 \\
\hline Ку 14 & PI 552477 & B & 43.5 & 295.6 \\
\hline Xanthi & PI 552780 & $\mathrm{O}$ & 43.6 & 205.7 \\
\hline TI 1605 & PI 415008 & B & 43.8 & 134.6 \\
\hline TI 835 & PI 118113 & $\mathrm{CF}$ & 46.2 & 343.5 \\
\hline N. sylvestris & PI 555570 & Sp. & 46.5 & 286.3 \\
\hline TI 1451 & PI 292205 & $\mathrm{CF}$ & 47.1 & 173.9 \\
\hline TI 1315 & PI 405603 & $\mathrm{O}$ & 47.2 & 175.6 \\
\hline TI 232 & PI 112227 & $\mathrm{CF}$ & 47.7 & 165.8 \\
\hline TI 1280 & PI 405600 & $\mathrm{O}$ & 48.7 & 235.5 \\
\hline N. knightiana & PI 555527 & Sp. & 48.7 & 531 \\
\hline TI 1555 & PI 378072 & $\mathrm{O}$ & 49 & 234.6 \\
\hline TI 109 & PI 105954 & $\mathrm{FC}$ & 50 & 236.5 \\
\hline NC 2326 & PI 552453 & $\mathrm{FC}$ & 50.7 & 209.8 \\
\hline TI 395 & PI 112783 & $\mathrm{O}$ & 52.2 & 187.5 \\
\hline TI 123 & PI 404976 & $\mathrm{CF}$ & 52.9 & 176.2 \\
\hline TI 1269 & PI 405590 & $\mathrm{O}$ & 53.1 & 192.7 \\
\hline TN 90 & PI 543792 & B & 53.7 & 473.9 \\
\hline TI 165 & PI 405002 & $\mathrm{CF}$ & 55.4 & 245.5 \\
\hline TI 1524 & PI 370290 & $\mathrm{FC}$ & 56.5 & 431.5 \\
\hline Conn. Broadleaf & PI 552619 & $\mathrm{CW}$ & 56.6 & 228.9 \\
\hline TI 501 & PI 113985 & $\mathrm{FC}$ & 56.8 & 266.4 \\
\hline Samsun & PI 552748 & $\mathrm{O}$ & 56.9 & 471.9 \\
\hline TI 1449 & PI 292203 & B & 57.2 & 313.1 \\
\hline N. rotundifolia & PI 555553 & Sp. & 57.4 & 684.4 \\
\hline Kavala & PI 552668 & $\mathrm{O}$ & 57.7 & 222.7 \\
\hline Havana 307 & PI 552348 & $\mathrm{CB}$ & 57.9 & 303.7 \\
\hline TI 79 & PI 67720 & $\mathrm{CF}$ & 57.9 & 184.5 \\
\hline N. glutinosa & PI 241768 & Sp. & 58 & 600.3 \\
\hline TI 716 & PI 116144 & FC & 58.6 & 290.3 \\
\hline N. velutina & PI 244638 & Sp. & 59.2 & 601.3 \\
\hline TI 88 & PI 404956 & $\mathrm{O}$ & 59.4 & 370 \\
\hline NC 297 & & $\mathrm{FC}$ & 59.7 & 427.1 \\
\hline TI 819 & PI 117674 & $\mathrm{~B}$ & 59.8 & 306.2 \\
\hline Speight G70 & PI 552497 & $\mathrm{FC}$ & 59.9 & 261.2 \\
\hline TI 1414 & PI 405662 & B & 60.1 & 289.3 \\
\hline TI 1379 & PI 286820 & $\mathrm{O}$ & 60.2 & 288.5 \\
\hline TI 672 & PI 114634 & $\mathrm{CF}$ & 60.8 & 434.2 \\
\hline MD 609 & PI 552452 & $\mathrm{M}$ & 61.3 & 304 \\
\hline TI 1577 & PI 372918 & $\mathrm{CF}$ & 62.7 & 290.2 \\
\hline Pennleaf 1 & PI 552403 & $\mathrm{CF}$ & 62.7 & 308.8 \\
\hline TI 1500 & PI 329206 & $\mathrm{FC}$ & 63.7 & 296.9 \\
\hline NC 95 & PI 552380 & $\mathrm{FC}$ & 63.7 & 300.7 \\
\hline TI 1222 & PI 405555 & $\mathrm{O}$ & 63.9 & 315.2 \\
\hline Florida 301 & PI 552629 & $\mathrm{CW}$ & 64.1 & 322.3 \\
\hline TI 1589 & PI 390128 & $\mathrm{CF}$ & 64.1 & 251.1 \\
\hline K 326 & PI 552505 & $\mathrm{FC}$ & 64.2 & 388 \\
\hline TI 1247 & PI 405577 & $\mathrm{O}$ & 65.3 & 282 \\
\hline TI 1241 & PI 405571 & $\mathrm{CF}$ & 65.7 & 117.1 \\
\hline Speight 168 & $\ldots$ & $\mathrm{FC}$ & 66.1 & 436.9 \\
\hline Havana 503 & PI 551281 & $\mathrm{CB}$ & 66.9 & 280.1 \\
\hline NC 2002 & $\ldots$ & B & 67.1 & 602.3 \\
\hline Clay 402 & $\ldots$ & B & 67.1 & 512.6 \\
\hline TI 158 & PI 404994 & $\mathrm{O}$ & 68.2 & 542 \\
\hline N. longiflora & PI 555531 & Sp. & 68.6 & 355 \\
\hline TI 186 & PI 112205 & $\mathrm{~B}$ & 69.8 & 345.4 \\
\hline TI 1279 & PI 405599 & $\mathrm{CF}$ & 69.9 & 317 \\
\hline TI 1462 & PI 304901 & $\mathrm{FC}$ & 70 & 762.8 \\
\hline$N$. langsdorffii & PI 42337 & Sp. & 70.9 & 583.4 \\
\hline \multirow[t]{2}{*}{ TI 1569} & PI 349332 & $\mathrm{~B}$ & 71 & 473.1 \\
\hline & & & \multicolumn{2}{|c|}{ (continued on next page } \\
\hline
\end{tabular}

${ }^{\text {a }}$ Plant introduction number.

${ }^{\mathrm{b}}$ Tobacco types are B, burley; CB, cigar binder; CF, cigar filler; CW, cigar wrapper; FC, flue-cured; $\mathrm{O}$, oriental; DFC, dark fire-cured; AC, air-cured; M, Maryland; and Sp., Nicotiana sp.

${ }^{c}$ Arcsine-transformed percentage of disease incidence.

d Area under the disease progress curve.

${ }^{\mathrm{e}}$ Least significant difference.

${ }^{f}$ Coefficient of variation. 
Table 1. (continued from preceding page)

\begin{tabular}{|c|c|c|c|c|}
\hline Genotype name & PI number ${ }^{a}$ & Type $^{\text {b }}$ & $\begin{array}{c}\text { Arcsine } \\
\text { incidence }(\%)^{\mathrm{c}}\end{array}$ & AUDPC $^{\mathbf{d}}$ \\
\hline McNair 944 & PI 552494 & $\mathrm{FC}$ & 72.7 & 461.5 \\
\hline TI 1330 & $\ldots$ & $\mathrm{CF}$ & 73.8 & 311.1 \\
\hline OX 414 & $\ldots$ & $\mathrm{FC}$ & 74.1 & 475 \\
\hline K 346 & PI 549110 & $\mathrm{FC}$ & 74.2 & 250.2 \\
\hline Coker 371 Gold & $\ldots$ & $\mathrm{FC}$ & 74.3 & 409.6 \\
\hline NC 5 & $\ldots$ & B & 74.5 & 349.1 \\
\hline $\mathrm{NC} 4$ & $\ldots$ & $\mathrm{B}$ & 74.8 & 565.6 \\
\hline TI 1306 & PI 241414 & $\mathrm{O}$ & 75.7 & 526.2 \\
\hline$N$. debneyi & PI 503323 & Sp. & 76.6 & 1008.1 \\
\hline TI 1224 & PI 405557 & FC & 77.1 & 448.2 \\
\hline $\mathrm{R} 7-11$ & $\ldots$ & B & 77.8 & 442.5 \\
\hline Speight H20 & $\ldots$ & $\mathrm{FC}$ & 77.9 & 229.6 \\
\hline Speight G28 & PI 551318 & $\mathrm{FC}$ & 83.1 & 530.3 \\
\hline TI 1512 & PI 370278 & $\mathrm{FC}$ & 83.4 & 436.2 \\
\hline TI 1480 & PI 405678 & $\mathrm{CF}$ & 85.6 & 436.8 \\
\hline TI 1558 & PI 355073 & $\mathrm{FC}$ & 86.4 & 374.4 \\
\hline NC 71 & $\ldots$ & $\mathrm{FC}$ & 88.1 & 406.7 \\
\hline TI 1616 & PI 418593 & $\mathrm{FC}$ & 90.1 & 265.2 \\
\hline Hicks & PI 552373 & $\mathrm{FC}$ & 91.5 & 389.5 \\
\hline Tom Rosson Madole & PI 552764 & DFC & 91.5 & 558.1 \\
\hline NC 72 & $\ldots$ & $\mathrm{FC}$ & 92.5 & 368 \\
\hline N. nudicaulis & PI 555540 & Sp. & 96.5 & 911.1 \\
\hline TI 1734 & $\ldots$ & $\mathrm{AC}$ & 97.8 & 715.8 \\
\hline TI 1518 & PI 370284 & $\mathrm{CF}$ & 97.9 & 624.1 \\
\hline N. repanda & PI 555552 & Sp. & 100.3 & 691.2 \\
\hline N. tomentosiformis & PI 555572 & Sp. & 100.3 & 651.5 \\
\hline Pennbel 69 & PI 552404 & $\mathrm{CF}$ & 103.3 & 239.4 \\
\hline TI 119 & PI 404974 & $\mathrm{CF}$ & 106.1 & 344.3 \\
\hline SA 1214 & $\ldots$ & $\mathrm{FC}$ & 122 & 1192.3 \\
\hline N. alata & PI 42334 & $\mathrm{Sp}$. & 150.2 & 1513.9 \\
\hline N. africana & PI 555472 & Sp. & 157.1 & 2815.5 \\
\hline Mean & $\ldots$ & $\ldots$ & 67.56 & 413.23 \\
\hline $\operatorname{LSD}(0.05)^{\mathrm{e}}$ & $\ldots$ & $\ldots$ & 42.47 & 435.26 \\
\hline $\mathrm{CV}(\%)^{\mathrm{f}}$ & $\ldots$ & $\ldots$ & 38.9 & 66.1 \\
\hline
\end{tabular}

Table 2. Comparison of genotypes with high and low stem rot incidence over multiple experiments

\begin{tabular}{lccccc}
\hline & & \multicolumn{4}{c}{ Incidence (\%) $^{\mathbf{a}}$} \\
\cline { 3 - 6 } Genotype & Class $^{\mathbf{b}}$ & Exp 1 & Exp 2 & Exp 3 & Average \\
\hline TI 1605 & 1 & 43.8 & NT & 38.4 & 41.1 \\
TI 1029 & 1 & 42.7 & 30.6 & 50.3 & 41.2 \\
TI 1311 & 1 & 39.7 & 49.2 & 56.0 & 48.3 \\
Xanthi & 1 & 43.6 & 50.1 & 57.2 & 50.3 \\
TI 1316 & 1 & 28.7 & 55.4 & 76.5 & 53.5 \\
Nicotiana rustica & 1 & 40.0 & 61.1 & 59.8 & 53.6 \\
TI 428 & 1 & 41.8 & 58.6 & 64.5 & 54.9 \\
Burley 21 & 1 & 42.0 & 77.0 & 63.7 & 60.9 \\
L8 & 1 & 42.7 & 91.4 & 62.7 & 65.6 \\
KY 14 & 1 & 43.5 & 87.3 & 66.6 & 65.8 \\
K326 & $\mathrm{m}$ & 64.2 & 82.7 & 82.9 & 76.6 \\
TI 1518 & $\mathrm{h}$ & 97.9 & 93.7 & 51.7 & 81.1 \\
TI 1734 & $\mathrm{h}$ & 97.8 & 82.8 & 70.2 & 83.6 \\
Hicks & $\mathrm{h}$ & 91.5 & 100.7 & 68.7 & 87.0 \\
TI 119 & $\mathrm{h}$ & 106.1 & 76.8 & 81.6 & 88.2 \\
Pennbel 69 & $\mathrm{h}$ & 103.3 & 97.2 & 67.8 & 89.4 \\
NC 72 & $\mathrm{h}$ & 92.5 & 110.1 & 82.1 & 94.9 \\
SA 1214 & $\mathrm{h}$ & 122.0 & 106.2 & 74.2 & 100.8 \\
N. alata & $\mathrm{h}$ & 150.2 & 107.3 & 107.3 & 121.6 \\
LSD (0.05) & $\ldots$ & $\ldots$ & 26.07 & 22.58 & $\ldots$ \\
CV (\%) & $\ldots$ & $\ldots$ & 19.63 & 20.20 & $\ldots$ \\
\hline
\end{tabular}

${ }^{a}$ Arcsine-transformed percentage of disease incidence for three experiments (Exp); NT $=$ not tested.

${ }^{\mathrm{b}}$ Initial classification. Genotypes with low (1), moderate (m), or high (h) stem rot disease incidence in the initial experiment.

${ }^{c}$ Least significant difference.

${ }^{\mathrm{d}}$ Coefficient of variation.

stem rot isolate, the pathogen would overcome and kill the small seedlings up to about two weeks after inoculation. After that time, little additional disease development occurred until about 35 days postinoculation (dpi), at which time stem rot lesions at the base of the stem began to develop. Disease incidence, which included the percentage of plants exhibiting seedling death and stem rot, was evaluated every other day for about 2.5 weeks post inoculation and then twice a week up to 42 dpi. For the target spot isolate, the pathogen also caused a small amount of seedling death up to about 2 weeks after inoculation. After this time, very little symptom development was observed until after 50 dpi, when target spot leaf lesions began to appear. The percentage of seedling death was evaluated every other day up to 2 to 2.5 weeks post inoculation and, at 8 weeks, the percentage of plants exhibiting target spot symptoms was evaluated.

Several accessions with the highest and lowest levels of disease were retested in additional experiments in order to confirm previous results. The stem rot test was repeated two more times with the selected accessions. Each of these tests consisted of three replications arranged in a randomized complete block design, with all three replications in a single phytotron chamber. The target spot test was repeated one additional time in an experiment with three replications. For the target spot isolate, several accessions were retested using a modification of the original protocol in order to evaluate resistance under higher disease pressure. In this test, the relative humidity was maintained at 90 to $98 \%$ by misting for $20 \mathrm{~s}$ every $5 \mathrm{~min}$ until $46 \mathrm{dpi}$, when target spot lesions first developed. Each replication was tented with plastic sheeting to prevent water from settling on the plants. The percentage of seedling death and plants with target spot was calculated as before. In addition, the diameter of each target spot lesion was measured to calculate the leaf area covered by target spot. The total area was divided by the number of living plants to determine the lesion area per plant.

In 2005, a heavy target spot outbreak was observed at the Border Belt Tobacco Research Station in Whiteville, NC. Official variety tests for flue-cured and burley cultivars had been planted at this site and both tests had significant disease. Target spot leaf lesions occurred most heavily on the lowest portions of the plants and declined toward the top the plants. The amount of leaf area with over $10 \%$ damage was recorded for plots of several burley and flue-cured cultivars over the three replications in these tests.

Statistical analysis. An arcsine transformation was performed on all percent incidence data before statistical analysis in order to improve homogeneity of variance. For the stem rot test, the area under the disease progress curve (AUDPC) was calculated using the formula $\sum_{i}^{n-1}\left[\left(y_{i}+\right.\right.$ $\left.\left.y_{i+1}\right) / 2\right]\left(t_{i+1}-t_{i}\right)$, where $n=$ number of disease assessments, $i=$ observations from 1 to $n, y_{\mathrm{i}}$ is the disease incidence on the $i$ th rating date, and $t_{i}$ is the day of the $i$ th rating $(1,13)$.

Analysis of variance was performed with PROC GLM and means were compared using Fisher's protected least sig- 
nificant difference test $(P=0.05)$ using SAS statistical software (SAS Institute, Inc., Cary, NC). Pearson's correlation coefficient was calculated to compare disease measurements in different tests.

\section{RESULTS}

Stem rot. Significant differences in overall disease incidence and AUDPC were observed between genotypes inoculated with the stem rot isolate of $R$. solani, although no accession was completely resistant (Table 1). The percentage of disease incidence ranged from 12.5 to $100 \%$ (28.7 to $157.1 \%$ after arcsine transformation). Percent incidence and AUDPC were well correlated in this test, with a correlation coefficient of $r=0.74(P<0.0001)$, but AUDPC had a much higher coefficient of variation. Among the U.S. cultivars, two burley cultivars, Burley 21 and Ky 14, were observed to have relatively low amounts of disease.

Because the least significant difference covered a wide range in this test, this experiment was repeated twice with several of the accessions with the lowest and highest disease ratings to determine whether there was a repeatable difference in the level of resistance of different cultivars (Table 2). There was a significant testgenotype interaction for disease incidence; therefore, the data for each test was analyzed separately. The disease incidence data from the first test was correlated with the second $(r=0.74, P=0.0005)$ and third test $(r=0.62, P=0.0045)$. The incidence for all three tests was averaged and compared with the original classifications. All of the accessions that were classified as having low disease incidence in the first experiment had a lower average disease incidence than the most susceptible accessions, although there was enough variation in individual tests so that some accessions did not match the original classification in all tests. The accession with the lowest disease incidence was TI 1605, which is a burley accession from Japan named Shiroenshu 202.

Target spot. Highly significant differences in disease incidence were observed among genotypes inoculated with an isolate of T. cucumeris that causes target spot (Table 3). The most common symptom produced by the target spot isolate was foliar target spot lesions; however, this disease also caused a low level of seedling death. The observed leaf area affected by target spot was low and target spot lesions developed late in the test. Disease incidence ranged from 6.2 to $97.9 \%$ (21.4 to $149.7 \%$ in arcsine-transformed data). TI 1605 also had the lowest disease incidence in this test, exhibiting no seedling death and a low percentage of seedlings with target spot. Among the cultivars tested, several burley cultivars had a relatively low disease incidence, including $\mathrm{TN} 90$, NC 2002, Burley 21, and Clay 402, whereas
Table 3. Reaction of Nicotiana accessions to target spot isolate of Thanatephorus cucumeris

\begin{tabular}{|c|c|c|c|}
\hline \multirow[b]{2}{*}{ Genotype name } & \multicolumn{3}{|c|}{ Arcsine (\%) } \\
\hline & Seedling death & Target spot & Total incidence \\
\hline TI 1605 & 0 & 21.4 & 21.4 \\
\hline Nicotiana nudicaulis & 9 & 20.9 & 26.3 \\
\hline$N$. africana & 22.6 & 12.5 & 28.7 \\
\hline N. glutinosa & 27 & 14.7 & 34.1 \\
\hline N. longiflora & 15.3 & 32.1 & 37.2 \\
\hline TN 90 & 10.2 & 34.5 & 37.6 \\
\hline N. knightiana & 21.4 & 24.3 & 38.3 \\
\hline NC 2002 & 7.3 & 37.1 & 39.3 \\
\hline TI 1524 & 5.1 & 34.6 & 39.7 \\
\hline N. debneyi & 12.4 & 31.7 & 40.5 \\
\hline TI 819 & 10.3 & 38.1 & 41.6 \\
\hline Clay 402 & 23.7 & 32 & 42.7 \\
\hline Burley 21 & 9 & 34.2 & 43.2 \\
\hline N. repanda & 22.9 & 32.1 & 45.1 \\
\hline TI 1449 & 10.3 & 43.2 & 45.7 \\
\hline TI 186 & 23.7 & 35.6 & 45.9 \\
\hline TI 1734 & 19.9 & 39.1 & 46.2 \\
\hline L8 & 19.2 & 40.3 & 47.6 \\
\hline TI 165 & 12.4 & 44 & 47.6 \\
\hline NC 4 & 13.2 & 41.8 & 48.5 \\
\hline TI 716 & 0 & 48.5 & 48.5 \\
\hline$N$. rotundifolia & 14.6 & 43.6 & 48.7 \\
\hline Ку 14 & 17.6 & 44.3 & 49.6 \\
\hline TI 1029 & 17.5 & 44.7 & 49.9 \\
\hline N. alata & 14.1 & 45.6 & 50.6 \\
\hline R7-11 & 14.6 & 44.8 & 50.8 \\
\hline N. sylvestris & 23.7 & 41.5 & 51.5 \\
\hline TI 428 & 24.8 & 44.8 & 52.7 \\
\hline Pennleaf 1 & 22.9 & 44.5 & 52.8 \\
\hline TI 672 & 19.6 & 46.2 & 52.9 \\
\hline TI 1569 & 5.1 & 52.2 & 53.4 \\
\hline$N$. tomentosiformis & 40.8 & 22.7 & 54.4 \\
\hline N. rustica & 30.9 & 33.5 & 54.5 \\
\hline TI 1555 & 5.1 & 53.7 & 54.9 \\
\hline TI 109 & 20.4 & 45.3 & 54.9 \\
\hline TI 1518 & 5.1 & 54.3 & 55.3 \\
\hline TI 835 & 23.7 & 46.3 & 55.5 \\
\hline NC 5 & 21.6 & 48.9 & 56.2 \\
\hline Speight 168 & 19.7 & 49.9 & 56.3 \\
\hline Tom Rosson Madole & 15.3 & 52.9 & 56.6 \\
\hline TI 1279 & 17.5 & 52.1 & 56.9 \\
\hline TI 1269 & 7.3 & 55.2 & 57.6 \\
\hline TI 1316 & 0 & 58.3 & 58.3 \\
\hline MD 609 & 5.1 & 58.1 & 59.2 \\
\hline Pennbel 69 & 20.5 & 47.3 & 59.4 \\
\hline TI 501 & 17.6 & 54.9 & 59.4 \\
\hline TI 232 & 9 & 56.9 & 60.3 \\
\hline Havana 307 & 0 & 61 & 61 \\
\hline Havana 503 & 0 & 62.4 & 62.4 \\
\hline TI 1247 & 23.2 & 54.6 & 62.5 \\
\hline McNair 944 & 12.4 & 59.2 & 62.5 \\
\hline SA 1214 & 43.4 & 40.1 & 62.9 \\
\hline TI 158 & 30.1 & 48 & 63.2 \\
\hline TI 1330 & 10.2 & 61.2 & 63.3 \\
\hline TI 1512 & 7.3 & 62.5 & 64.6 \\
\hline Speight G28 & 24.2 & 54.5 & 64.7 \\
\hline Conn. Broadleaf & 10.2 & 62.6 & 64.7 \\
\hline TI 1306 & 12.4 & 62.2 & 65.3 \\
\hline TI 119 & 26 & 53.5 & 65.5 \\
\hline TI 1500 & 17.5 & 60.6 & 65.7 \\
\hline TI 1222 & 0 & 65.8 & 65.8 \\
\hline TI 1558 & 5.1 & 64.8 & 65.8 \\
\hline TI 123 & 19.2 & 61.1 & 66.5 \\
\hline TI 1414 & 10.2 & 64.7 & 66.8 \\
\hline TI 1315 & 0 & 67.9 & 67.9 \\
\hline TI 79 & 5.1 & 66.9 & 67.9 \\
\hline TI 1241 & 12.4 & 65.2 & 68.3 \\
\hline TI 1462 & 10.2 & 66.8 & 68.9 \\
\hline TI 1451 & 22 & 61.4 & 69 \\
\hline \multirow{2}{*}{ N. velutina } & 24.1 & 55.3 & 69.4 \\
\hline & & & inued on next $p$ \\
\hline
\end{tabular}

\footnotetext{
${ }^{a}$ Least significant difference.
}

${ }^{b}$ Coefficient of variation. 
Table 3. (continued from preceding page)

\begin{tabular}{|c|c|c|c|}
\hline \multirow[b]{2}{*}{ Genotype name } & \multicolumn{3}{|c|}{ Arcsine (\%) } \\
\hline & Seedling death & Target spot & Total incidence \\
\hline TI 1589 & 0 & 70 & 70 \\
\hline Kavala & 0 & 70.5 & 70.5 \\
\hline TI 1224 & 7.3 & 69 & 71.1 \\
\hline N. langsdorffii & 22.6 & 63.1 & 71.1 \\
\hline TI 1616 & 19.2 & 67.7 & 73 \\
\hline Speight G70 & 0 & 73.1 & 73.1 \\
\hline TI 1379 & 7.3 & 71.2 & 73.3 \\
\hline Coker 371 Gold & 26 & 65.7 & 75.1 \\
\hline NC 71 & 17.5 & 71.8 & 76.2 \\
\hline Xanthi & 12.4 & 73.2 & 76.4 \\
\hline Florida 301 & 10.2 & 74.3 & 76.9 \\
\hline TI 1577 & 12.4 & 75.4 & 78.5 \\
\hline K 346 & 20.6 & 72.2 & 78.6 \\
\hline TI 88 & 29.7 & 69.7 & 80 \\
\hline Hicks & 25.1 & 71.8 & 80.7 \\
\hline TI 1311 & 28.8 & 70.5 & 83 \\
\hline K 326 & 10.2 & 77.7 & 83.8 \\
\hline Samsun & 45.4 & 63.6 & 84.9 \\
\hline TI 1480 & 27.4 & 77.4 & 85.5 \\
\hline NC 297 & 12.4 & 83.5 & 86.6 \\
\hline TI 1280 & 12.5 & 83.8 & 87 \\
\hline NC 95 & 7.7 & 88.6 & 96.3 \\
\hline NC 2326 & 10.2 & 91.9 & 98.1 \\
\hline $\mathrm{NC} 72$ & 12.4 & 95 & 98.2 \\
\hline Speight H20 & 23.8 & 89.4 & 113.2 \\
\hline OX 414 & 24.8 & 112.2 & 127.9 \\
\hline TI 395 & 15.3 & 137.8 & 149.7 \\
\hline Mean & 15.1 & 55.9 & 62.4 \\
\hline $\operatorname{LSD}(0.05)^{\mathrm{a}}$ & 21.2 & 23.3 & 22.8 \\
\hline $\mathrm{CV}(\%)^{\mathrm{b}}$ & 100.1 & 29.6 & 26 \\
\hline
\end{tabular}

Table 4. Comparison of selected genotypes infected with target spot in a high-humidity environment

\begin{tabular}{lccccc}
\hline Genotype & Class $^{\mathbf{a}}$ & $\begin{array}{c}\text { Seedling } \\
\text { death }^{\mathbf{b}}\end{array}$ & $\begin{array}{c}\text { Target } \\
\text { spot }^{\mathbf{b}}\end{array}$ & $\begin{array}{c}\text { Total } \\
\text { incidence }^{\mathbf{b}}\end{array}$ & $\begin{array}{c}\text { Lesion area } \\
\text { per }_{\text {plant }}\left(\mathbf{c m}^{\mathbf{2}}\right)\end{array}$ \\
\hline TI 1605 & 1 & 7.0 & 17.0 & 20.0 & 0.01 \\
NC 2002 & 1 & 30.6 & 6.9 & 32.4 & 0.02 \\
NC 2326 & $\mathrm{h}$ & 34.9 & 13.7 & 38.5 & 0.02 \\
TN 90 & 1 & 32.8 & 12.1 & 38.8 & 0.06 \\
TI 1311 & $\mathrm{h}$ & 35.0 & 20.9 & 41.7 & 0.08 \\
NC 95 & $\mathrm{h}$ & 34.9 & 24.1 & 43.1 & 0.35 \\
K 326 & $\mathrm{h}$ & 23.2 & 32.5 & 43.6 & 0.27 \\
Burley 21 & 1 & 39.6 & 16.6 & 44.7 & 0.10 \\
NC 4 & 1 & 35.6 & 29.2 & 47.2 & 0.18 \\
TI 819 & 1 & 37.0 & 24.1 & 47.9 & 0.09 \\
NC 72 & $\mathrm{h}$ & 32.9 & 23.4 & 48.4 & 0.19 \\
Hicks & $\mathrm{h}$ & 37.0 & 32.4 & 51.6 & 0.52 \\
K 346 & $\mathrm{h}$ & 47.7 & 24.3 & 58.5 & 0.06 \\
TI 1280 & $\mathrm{h}$ & 36.4 & 43.4 & 58.8 & 0.29 \\
KY 14 & 1 & 55.4 & 9.8 & 59.7 & 0.01 \\
L8 & 1 & 56.0 & 16.6 & 60.2 & 0.19 \\
Speight H-20 & $\mathrm{h}$ & 45.6 & 35.9 & 62.0 & 0.28 \\
TI 1316 & $\mathrm{m}$ & 34.0 & 47.0 & 65.0 & 0.72 \\
TI 1480 & $\mathrm{h}$ & 55.8 & 25.6 & 66.4 & 0.18 \\
OX 414 & $\mathrm{h}$ & 55.6 & 40.5 & 74.2 & 0.44 \\
LSD (0.05) & $\ldots$ & 23.0 & NS & NS & NS \\
\hline
\end{tabular}

a Initial classification. Genotypes with high (h) and low (1) disease incidence in initial experiment.

b Data shown is arcsine-transformed percentage of disease incidence.

${ }^{\mathrm{c}}$ Least significant difference; NS = no significant difference.

most of the flue-cured cultivars had a high disease incidence.

The target spot experiment also was repeated with several accessions that had the highest and lowest disease incidence (Table 4). Because the leaf area affected by target spot lesions was small, the experiment was modified to provide a high-humidity environment that was thought to increase the development of target spot. However, this environment was very favorable for disease development and resulted in a higher frequency of seedling death. In this environment, there was a significant difference between cultivars for seedling death, with TI 1605 exhibiting significantly less seedling death than almost all the other accessions. There were no significant differences for target spot incidence, total incidence, or lesion area per plant in this experiment.
Table 5. Field observations of target spot severity in burley and flue-cured cultivars

\begin{tabular}{lcc}
\hline Type, cultivar & $\begin{array}{c}\text { Leaf area } \\
\text { affected (\%) }\end{array}$ & $\begin{array}{c}\text { Standard } \\
\text { deviation }\end{array}$ \\
\hline Burley & & \\
NC 2002 & 30.0 & 0.0 \\
TN 90 & 32.5 & 5.0 \\
NC 5 & 32.5 & 5.0 \\
Flue-cured & & \\
K 326 & 36.7 & 8.2 \\
K 346 & 43.3 & 5.0 \\
NC 71 & 40.0 & 10.0 \\
\hline
\end{tabular}

The lower incidence of target spot symptoms in seedlings of burley cultivars that was observed in the first experiment was not confirmed in the second growthchamber experiment. However, an opportunity to compare target spot symptoms in mature field-grown plants occurred in 2005, when a heavy target spot outbreak was observed at the Border Belt Tobacco Research Station in Whiteville, NC (Table 5). Official variety tests for flue-cured and burley cultivars had been planted in adjacent plots, and six cultivars in this test also had been tested in the growth-chamber experiment. Although the burley cultivars had a slightly lower level of leaf area affected, this level was not substantially different compared with the flue-cured cultivars.

\section{DISCUSSION}

Significant differences in disease incidence and AUDPC were observed among tobacco genotypes evaluated for resistance to stem rot. Variation in disease development required that accessions be tested several times to identify genotypes that were consistently resistant. Several accessions, including TI 1605, TI 1029, TI 1311 , and 'Xanthi,' consistently had low disease incidence in multiple tests. Among U.S. cultivars, the burley cvs. Burley 21 and Ky 14 had a low average disease incidence, but this was not consistently observed in repeated tests. In contrast, the flue-cured cvs. Hicks and NC 72 had a high incidence of disease.

Partial resistance to target spot was not consistently identified in these tests. Although there were significant genotype differences in seedling death, target spot, and total incidence in the initial observations, these differences were not observed when the test was conducted under a highhumidity environment designed to increase disease severity. Although the development of target spot lesions was increased, this environment also substantially increased seedling death. One genotype, TI 1605 , had a significantly lower incidence of seedling death than almost all of the other accessions. TI 1605 also was one the genotypes that was most resistant to the stem rot isolate.

Based on the initial growth-chamber experiment, it appeared that several burley 
cultivars had a significantly lower target spot disease incidence than flue-cured cultivars. However, this observation was not confirmed in the second growthchamber experiment or in a natural field infection. Shew and Melton noted that differences in target spot disease severity have been observed among different genotypes in field and growth-chamber tests, but that this level of resistance was not high enough to provide control of the disease when environmental conditions were favorable (17). The results from the second growth-chamber experiment appear to confirm this observation. Csinos and Stephenson reported that several commonly used flue cultivars, including NC 71, NC 72, K326, Coker 371-Gold, Speight G-70, and Speight G-168, had low to moderate disease levels in a natural field epidemic compared with several breeding lines that had not yet been accepted as cultivars (3). These cultivars did not appear to have resistance to target spot in the growthchamber experiments or in the field epidemic we observed. However, the breeding lines that were present in their test were not available for our experiment, and may represent highly susceptible genotypes.

It also is possible that there are differences between the North Carolina and Georgia target spot populations which might account for the lower disease levels observed in the Georgia epidemic. However, genetic diversity in tobacco target spot isolates collected throughout North Carolina has been examined by comparison of molecular marker profiles and somatic compatibility (2). This study showed that the tobacco isolates had lower diversity compared with isolates from potato, and that four clones represented $22 \%$ of the total population. Two of these clones were recovered in more than one field in widely separated (by $220 \mathrm{~km}$ ) counties. The relatively low diversity of tobacco isolates over a widespread area in North Carolina suggests that target spot populations across the southeastern tobaccogrowing region are similar.

Partial resistance to $R$. solani diseases has been described in several crops, and resistance often is expressed in the form of a reduction in disease severity or rate of infection. Two dominant major resistance genes have been identified as conferring partial resistance to $R$. solani in rice (10). In peanut, it has been suggested that some genotypes may have partial resistance due to an erect growth habit that allows more rapid leaf drying and reduced soil contact. However, resistance also has been identified in genotypes with a spreading growth habit and, for these genotypes, it was hypothesized that differences in resistance were due to physiological or biochemical responses (4). In our study, all of the seedlings were grown in a high-density hydroponic environment; therefore, the resistance observed could not be explained by differences in plant architecture.

This study identified genotypes with partial resistance to damping-off and stem rot caused by an AG-4 stem rot isolate of $R$. solani but did not identify resistance to foliar symptoms caused by an AG-3 target spot isolate. Of particular interest is the accession TI 1605, which appears to be resistant to damping-off and stem rot symptoms caused by both isolates. The accessions identified in this work may be useful as sources of disease resistance to $R$. solani in tobacco-breeding programs. In addition, there are a large number of accessions that could be tested in the future.

\section{ACKNOWLEDGMENTS}

We thank the North Carolina Tobacco Research Commission for financial support for this project.

\section{LITERATURE CITED}

1. Campbell, C. L., and Madden, L. V. 1990. Introduction to Plant Disease Epidemiology. John Wiley \& Sons, New York.

2. Ceresini, P. C., Shew, H. D., Vilgalys, R. J., and Cubeta, M. A.2002. Genetic diversity of Rhizoctonia solani AG-3 from potato and tobacco in North Carolina. Mycologia 94:437449.

3. Csinos, A. S., and Stephenson, M. G. 1999. Evaluation of fungicides and tobacco cultivar resistance to Rhizoctonia solani incited target spot, damping off and sore shin. Crop Prot. 18:373-377.

4. Francke, M. D., Brenneman, T. B., and Holbrook, C. C. 1999. Identification of resistance to Rhizoctonia limb rot in a core collection of peanut germplasm. Plant Dis. 83:944-948.

5. Gutierrez, W. A., Shew, H. D., and Melton, T. A. 1997. Sources of inoculum and management for Rhizoctonia solani damping-off on tobacco transplants under greenhouse conditions. Plant Dis. 81:604-606.

6. Lewis, R. S., and Nicholson, J. S. 2007. As- pects of the evolution of Nicotiana tabacum L. and the status of the United States Nicotiana Germplasm Collection. Genet. Resour. Crop Evol. 54:727-740.

7. Lucas, G. B. 1975. Diseases of Tobacco, 3rd ed. Biological Consulting Associates, Raleigh, NC.

8. Mina, A., Gutierrez, W. A., and Broadwell, A. 2006. Extension-Research Flue-Cured and Burley Tobacco Pathology Program Annual Report. North Carolina Cooperative Extension Service, North Carolina State University, Raleigh.

9. Montoya, C. A., Beaver, J. S., Rodriguez, P. N., Miklas, P. N., and Godoy-Lutz, G. 1997. Heritability of resistance of web blight in five common bean populations. Crop Sci. 37:780783.

10. Pan, X. B., Rush, M. C., Sha, X. Y., Xie, Q. J., Linscombe, S. D., Stetina, S. R., and Oard, J. H. 1999. Major gene, nonallelic sheath blight resistance from the rice cultivars Jasmine 85 and Tequing. Crop Sci. 39:338-346.

11. Pascual, C. B., Raymundo, A. D., and Hyakumachi, M. 2000. Resistance of sorghum line CS 621 to Rhizoctonia solani AG1-IA and other sorghum pathogens. J. Gen. Plant Pathol 66:23-29.

12. Scholten, O. E., Panella, L. W., De Bock, T. S. M., and Wouter, L. 2001. A greenhouse test for screening sugar beet (Beta vulgaris) for resistance to Rhizoctonia solani. Eur. J. Plant Pathol. 107:161-166.

13. Shaner, G., and Finney, R. E. 1977. The effect of nitrogen fertilization on the expression of slow mildewing resistance in Knox wheat. Phytopathology 67:1051-1056.

14. Shew, H. D. 1991. Target spot. In: Compendium of Tobacco Diseases. H. D. Shew and G. B. Lucas, eds. The American Phytopathological Society, St. Paul, MN.

15. Shew, H. D. 1991. Sore shin and damping-off. In: Compendium of Tobacco Diseases. H. D. Shew and G. B. Lucas, eds. The American Phytopathological Society, St. Paul, MN

16. Shew, H. D., and Main, C. E. 1990. Infection and development of target spot of flue-cured tobacco caused by Thanatephorus cucumeris. Plant Dis. 74:1009-1013.

17. Shew, H. D., and Melton, T. A. 1995. Target spot of tobacco. Plant Dis. 79:6-11.

18. Smith, W. D., Fisher, L. R., and Spears, J. F. 2003. Transplant production in the float system. In: Flue-Cured Tobacco Information. W. D. Smith, ed. North Carolina Cooperative Extension Service, Raleigh.

19. Sneh, B., Jabaji-Hare, S., Neate, S., and Dijst, G., eds. 1996. Rhizoctonia Species: Taxonomy, Molecular Biology, Ecology, Pathology, and Disease Control. Kluwer Academic Publishers, Dordrecht, The Netherlands.

20. Stevens Johnk, J. S., Jones, R. K., Shew, H. D., and Carling, D. E. 1993. Characterization of populations of Rhizoctonia solani AG-3 from potato and tobacco. Phytopathology 83:854858. 